\section{Avaliação das informações do Sistema de Informações sobre Nascidos Vivos (SINASC), Brasil}

\section{Evaluation of data from the Brazilian Information System on Live Births (SINASC)}

Evaluación de la información del Sistema de
Información sobre Nacidos Vivos (SINASC), Brasil

\author{
Célia Landmann Szwarcwald 1 \\ Maria do Carmo Leal 2 \\ Ana Paula Esteves-Pereira 2 \\ Wanessa da Silva de Almeida 1 \\ Paulo Germano de Frias 3 \\ Giseli Nogueira Damacena 1 \\ Paulo Roberto Borges de Souza Júnior 1 \\ Narayani Martins Rocha 1 \\ Priscila Melissa Honorato Mullachery ${ }^{4}$
}

\section{Resumo}

Neste trabalho, avaliam-se quantitativa e qualitativamente as informações do Sistema de Informações sobre Nascidos Vivos (SINASC). A cobertura das informações por município foi estimada pela razão entre nascidos vivos informados e estimados. Para avaliação da qualidade das informações do SINASC, relacionou-se o sistema à base de dados do estudo Nascer no Brasil, 20112012, e foram estimados coeficientes kappa de concordância. Em 2013, a cobertura das informações foi alta e homogênea em todas as Unidades da Federação. Entretanto, a análise por município brasileiro apresentou maior heterogeneidade espacial. Quanto à qualidade de preenchimento das informações do SINASC, os coeficientes kappa de concordância foram estatisticamente diferentes de zero para todas as variáveis testadas ( $p<0,001)$, e as distribuições marginais para todas as variáveis consideradas foram semelhantes nas duas bases de dados. A idade gestacional foi a variável que mostrou pior concordância, com valor de kappa de 0,461. O indicador que descreve as inconsistências, medido pela soma do quadrado das diferenças entre os percentuais de prematuridade informados e esperados por faixa de peso ao nascer, teve o valor mais alto na Região Norte e o menor na Região Sul, apontando para desigualdades geográficas na mensuração da idade gestacional.

Nascimento Vivo; Sistemas de Informações em Saúde; Gestão da Qualidade

\author{
Correspondência \\ W. S. Almeida \\ Instituto de Comunicação e Informação Científica e Tecnológica \\ em Saúde, Fundação Oswaldo Cruz. \\ Av. Brasil 4365, Biblioteca de Manguinhos, 2o andar, sala 225, \\ Rio de Janeiro, RJ 21045-360, Brasil. \\ wanessa.almeida@gmail.com \\ 1 Instituto de Comunicação e Informação Científica e \\ Tecnológica em Saúde, Fundação Oswaldo Cruz, Rio de Janeiro, \\ Brasil. \\ 2 Escola Nacional de Saúde Pública Sergio Arouca, Fundação \\ Oswaldo Cruz, Rio de Janeiro, Brasil. \\ 3 Instituto de Medicina Integral Fernando Figueira, Recife, \\ Brasil. \\ 4 College of Global Public Health, New York University, New \\ York, U.S.A.
}




\section{Introdução}

Os dados provenientes dos certificados de nascimento são considerados de relevância em todo o mundo, tanto para a construção de indicadores de saúde como também para o desenvolvimento de programas de assistência materno-infantil 1. No Brasil, até a década de 1990, as informações referentes aos nascimentos eram provenientes apenas das Estatísticas do Registro Civil, de responsabilidade do Instituto Brasileiro de Geografia e Estatística (IBGE) 2. Contudo, sendo o registro civil um ato fundamentalmente jurídico, as informações referiam-se, basicamente, à comprovação legal do evento, sem prover dados importantes para a área de saúde, como as condições da criança ao nascer. A experiência de vários países mostrava que as melhores informações sobre as condições de nascimento eram geradas nos serviços de saúde onde ocorriam os nascimentos 3 .

Diante da importância do conhecimento das características do recém-nascido e da mãe, assim como da gravidez e do parto para o monitoramento das ações de saúde e para a formulação de políticas públicas, o Ministério da Saúde implantou, em 1990, o Sistema de Informações sobre Nascidos Vivos (SINASC). O sistema tem como base a declaração de nascido vivo (DNV), documento cuja emissão é obrigatória no serviço de saúde onde ocorreu o parto. Diferentemente do sistema de nascimentos do Registro Civil, cujo objetivo principal é a contagem do número de registros de nascimentos, o SINASC tem como propósito caracterizar as condições da gestação, do parto e do nascimento.

O primeiro modelo de DNV continha dados sobre o cartório de registro e o local de ocorrência do nascimento, informações sobre o recém-nascido (data do nascimento, sexo, peso ao nascer, índice de Apgar) e sobre a gravidez (duração da gestação, tipo de gravidez e tipo de parto), características da mãe do nascido vivo (nome, idade, grau de instrução, município de residência e filhos tidos) e nome do pai. Ao longo dos anos, foram realizadas algumas modificações na DNV original, com a introdução de novas variáveis (como raça/cor e presença de anomalia congênita), alterações nas categorias de análise (número de consultas pré-natal) e eliminação de outras (como o nome do pai), bem como mudanças na forma de mensuração de algumas questões, como o cálculo da idade gestacional 3 .

O SINASC está implantado em todos os estados brasileiros, e as informações são divulgadas pelo sítio da Internet do DATASUS - Ministério da Saúde (http://tabnet.datasus.gov.br/cgi/def tohtm.exe?sinasc/cnv/nvuf.def), sendo disponíveis, publicamente, por município, sem necessidade de autorização prévia para o seu uso. O sistema representa uma fonte de dados de inestimável valor para orientar as políticas de atenção à gestante e ao recém-nascido nos níveis municipal, estadual e nacional 4.

Vários estudos de avaliação das informações do SINASC estão disponíveis atualmente na literatura científica 4,5,6,7,8. Os artigos que abordam a qualidade das informações apontam para a baixa confiabilidade de alguns campos da declaração de nascido vivo e indicam a necessidade de avaliação específica para o maior controle de qualidade e otimização na implantação e uso de todas as informações do sistema 4,8. O estudo Nascer no Brasil, inquérito nacional sobre parto e nascimento 9, trouxe igualmente insumos importantes para a avaliação da qualidade das informações do SINASC.

O presente artigo teve como objetivos propor uma metodologia para estimação da cobertura das informações de nascidos vivos por município brasileiro e avaliar a qualidade das informações do SINASC a partir do relacionamento do sistema com a base de dados do estudo Nascer no Brasil e da análise de confiabilidade, medida pela concordância 10. Pretende, assim, apoiar a elaboração de critérios para avaliação das informações sobre nascidos vivos, no que se refere à análise quantitativa e qualitativa no preenchimento dos dados. 


\section{Métodos}

\section{Estimação da cobertura das informações do SINASC}

Para caracterizar o nível de cobertura das informações de nascidos vivos por município, no ano de 2013, calculou-se a razão entre nascidos vivos informados e estimados em todos os municípios brasileiros 11 . O número estimado de nascidos vivos $\left(N V_{\text {estimado }}\right)$ foi baseado na projeção da população de menores de um ano:

$$
N V_{\text {estimado }}=\frac{\text { População menor de 1ano }}{\left(1-\left(0,5 \times q_{0}\right)\right)}
$$

onde $\mathrm{q}_{0}$ representa o coeficiente de mortalidade infantil (CMI) da Unidade da Federação (UF) na qual está localizado o município. Na equação 1, foi utilizada a projeção da população de menores de um ano para o ano de 2013, realizada pelo IBGE, e o CMI estimado por UF para o ano de 201212.

O nível de cobertura das informações de nascidos vivos foi estimado por:

$$
\text { Cobertura de } N V(\%)=\frac{\text { mínimo }\left(N V_{\text {informado }}, N V_{\text {estimado }}\right)}{N V_{\text {estimado }}} \times 100
$$

em que o número informado de nascidos vivos $\left(N V_{\text {informado }}\right)$ foi calculado no triênio 2012-2014, considerando-se a média dos dados de nascidos vivos informados nos anos de 2012, 2013 e 2014, para dar maior estabilidade ao indicador nos municípios de pequeno porte populacional.

Quando a proporção calculada pela equação (2) é maior ou igual a 90\%, o número corrigido de nascidos vivos é igual ao número médio informado no SINASC no triênio 2012-2014. No caso de proporção inferior a 90\%, o número corrigido de nascidos vivos é dado pelo número estimado de nascidos vivos. O número de nascidos vivos corrigido por UF, no ano de 2013, foi obtido pela soma dos nascidos vivos corrigidos dos municípios daquela UF, e a cobertura das informações de nascidos vivos por UF foi estimada pela razão entre o número informado e corrigido de nascidos vivos.

A cobertura por UF foi correlacionada à proporção de óbitos fetais com peso maior ou igual a 2.500g entre os óbitos fetais informados no Sistema de Informações sobre Mortalidade (SIM), indicador de possível classificação incorreta de nascidos vivos como natimortos.

\section{Avaliação da qualidade das informações do SINASC}

Nesta etapa, foi feito o relacionamento entre as informações do SINASC (2011-2012) e a base de informações coletadas entre puérperas no estudo Nascer no Brasil, estudo seccional por amostragem probabilística de base hospitalar e abrangência nacional, realizado entre fevereiro de 2011 e outubro de 2012, em 266 maternidades localizadas em 191 municípios brasileiros 13.

Para a realização do relacionamento entre as bases de dados, foi utilizado o programa SPSS (https://www.ibm.com/). Dos campos do tipo nominal, compostos de caracteres, foram retirados acentos, cedilhas, algarismos e outros símbolos. Foi feita, igualmente, a conversão de letras minúsculas em maiúsculas. O campo da DNV na base do estudo Nascer no Brasil foi ajustado para ter oito dígitos, como apresentado no SINASC.

O emparelhamento das duas bases de dados foi feito por meio de variáveis-chave, já padronizadas em relação ao formato de apresentação e de escrita. O SPSS não faz o relacionamento probabilístico e apenas disponibiliza os pares que são coincidentes para todas as variáveis-chave utilizadas.

Três arquivos foram submetidos ao processo de relacionamento: os bancos de dados do SINASC de 2011 e 2012 e também o banco de dados com os registros das puérperas entrevistadas no estudo Nascer no Brasil. Na primeira etapa de relacionamento dos bancos, o número da DNV foi utilizado como chave primária. Após esse procedimento, os casos relacionados foram confirmados pelo nome da mãe.

Os casos não relacionados passaram por uma segunda etapa de pareamento, utilizando uma combinação entre o nome da mãe e a data do parto como variável-chave. As demais variáveis de identificação, como município de residência e código do estabelecimento onde ocorreu o parto, foram usadas para assegurar o emparelhamento correto dos casos. Terminado o relacionamento, foram 
descartados os casos não pareados ou dúbios, para evitar erros de interpretação da qualidade dos dados do SINASC.

Para a análise estatística, a base relacionada de dados, incluindo as informações do SINASC e do Projeto Nascer, foi calibrada para representar o Brasil 13. Para verificação da concordância de preenchimento das informações comuns aos dois bancos, foram analisadas as seguintes variáveis: faixa de idade ( $<15 ; 15-19 ; 20-24 ; 25-29 ; 30-34 ; 35-39 ; 40-44 ; \geq 45$ ), anos de estudo da mãe (nenhum; 1-3; 4-7; $8-11 ; \geq 12)$, peso ao nascer $(<1.500 \mathrm{~g} ; 1.500 \mathrm{~g}-1.999 \mathrm{~g} ; 2.000 \mathrm{~g}-2.499 \mathrm{~g} ; 2.500 \mathrm{~g}-2.999 \mathrm{~g} ; 3.000 \mathrm{~g}-3.499 \mathrm{~g}$; $3.500 \mathrm{~g}-3.999 \mathrm{~g} ; \geq 4.000 \mathrm{~g}$ ), idade gestacional (em semanas) ( $<27 ; 28-31 ; 32-36 ; 37-41 ; \geq 42)$, número de consultas pré-natal (nenhuma; 1-3; 4-6; $\geq 7$ ), tipo de gestação (única; gemelar ou mais), tipo de parto (vaginal; cesáreo) e Apgar no primeiro e quinto minutos ( 0 a 3; 4-6; 7; 8-10), utilizando-se o coeficiente kappa de concordância e respectivo teste de significância.

As análises de peso ao nascer e prematuridade foram realizadas por UF, para captar inconsistências geográficas no preenchimento dessas variáveis. Além disso, para avaliar a consistência das informações de idade gestacional do SINASC de acordo com o peso ao nascer por Grande Região, os dados do estudo Nascer no Brasil foram considerados como o padrão. A inconsistência das informações de idade gestacional do SINASC 2013 foi investigada por meio de gráfico para a visualização da distância entre os percentuais de prematuridade informados por Grande Região e o padrão e foi estabelecida pela soma do quadrado das diferenças (SQE) entre os percentuais informados e esperados.

\section{Resultados}

Na Tabela 1, são apresentadas as coberturas das informações de nascidos vivos por UF, que se mostraram altas e homogêneas, superiores a $90 \%$, exceto no Maranhão $(84,3 \%)$ e na Bahia $(88,5 \%)$. Ao se analisar, entretanto, as coberturas por município brasileiro, observa-se maior heterogeneidade espacial (Figura 1). No Amazonas, Pará e Maranhão, mais de 10\% dos municípios têm coberturas inferiores a $60 \%$.

Ainda na Tabela 1, apresenta-se a proporção de óbitos fetais com peso maior ou igual a $2.500 \mathrm{~g}$ entre os óbitos fetais informados ao SIM. As maiores proporções são encontradas nos estados do Maranhão (39,5\%), Amazonas (37\%) e Alagoas (33,6\%), ao passo que as menores são em São Paulo $(21,1 \%)$, Minas Gerais (22,2\%), Rio de Janeiro (24,4\%) e Rio Grande do Sul (24,5\%). A correlação entre esse indicador e a cobertura de nascidos vivos foi inversa $(-0,63)$ e estatisticamente significativa $(\mathrm{p}<0,001)$.

Adicionalmente, na Tabela 1, estão apresentados os percentuais de baixo peso ao nascer por UF, subdivididos por faixas (menor do que 1.500 , e de $1.500 \mathrm{~g}$ a $2.499 \mathrm{~g}$ ), e os percentuais de prematuridade, calculados mediante os dados do SINASC 2013, excluindo-se os casos com informações ignoradas. Em relação ao baixo peso ao nascer, percebem-se os menores percentuais nos estados de pior nível socioeconômico, com amplitude de variação de 6,5\% em Rondônia a 9,6\% no Distrito Federal. Diferenças nítidas são encontradas, igualmente, nos percentuais de nascidos vivos com peso inferior a $1.500 \mathrm{~g}$, com estados do Norte apresentando percentuais menores do que $1 \%$, ao passo que os do Sudeste indicam percentuais próximos a 1,5\%. Quanto aos percentuais de prematuridade, variaram de $8,9 \%$ em Sergipe a 15,2\% em Roraima. No Rio Grande do Norte, no Acre e no Pará, os percentuais de nascidos vivos prematuros ultrapassaram 13\%. As demais UF apresentaram percentuais próximos à média nacional, de 11,9\%, no ano de 2013.

No que se refere ao relacionamento das bases de dados do SINASC e do estudo Nascer no Brasil para avaliação da qualidade das informações de nascidos vivos, um resumo do procedimento de linkage está apresentado na Figura 2. A base de dados do estudo Nascer no Brasil contém 24.197 casos: 22.543 do ano de 2011 e 1.654 do ano de 2012. O banco SINASC 2011 tem 2.913 .160 casos, e o banco SINASC 2012 conta com 2.905.789 casos. Após a padronização da variável referente ao número da DNV no banco Nascer no Brasil (apresentação em oito caracteres, conforme SINASC), foram obtidas 20.257 DNV preenchidas e 3.940 não identificadas. Foram eliminados da análise 174 casos do banco Nascer no Brasil por conta de duplicidade de DNV. Dos 20.083 casos com DNV válida, 19.880 foram relacionados ao SINASC pela DNV. Esses casos passaram por uma confirmação pelo nome da mãe e, desse quantitativo, 19.606 foram confirmados. Os casos que não foram relacionados na primeira 
Tabela 1

Cobertura das informações de nascidos vivos, percentual de natimortos com peso igual ou superior a 2.500g, proporção de nascidos vivos com baixo peso ao nascer e proporção de prematuridade, por Unidade da Federação (UF), Brasil, 2012-2014.

\begin{tabular}{|c|c|c|c|c|c|c|}
\hline \multirow[t]{2}{*}{ UF } & \multirow{2}{*}{$\begin{array}{l}\text { Cobertura das informações } \\
\text { de nascidos vivos (\%) * }\end{array}$} & \multirow{2}{*}{$\begin{array}{l}\text { Natimortos } \geq \\
2.500 \mathrm{~g}(\%) * *\end{array}$} & \multicolumn{3}{|c|}{ Peso ao nascer (nascidos vivos) *** } & \multirow[t]{2}{*}{ Prematuridade $(\%) * * *$} \\
\hline & & & $<1.500 \mathrm{~g}$ & $1.500-2.499 \mathrm{~g}$ & $<2.500 \mathrm{~g}$ & \\
\hline Rondônia & 92,6 & 27,8 & 0,93 & 5,6 & 6,5 & 10,8 \\
\hline Acre & 97,5 & 32,3 & 1,21 & 6,6 & 7,8 & 13,5 \\
\hline Amazonas & 91,2 & 37,0 & 1,03 & 6,7 & 7,8 & 12,6 \\
\hline Roraima & 98,6 & 33,3 & 0,95 & 6,6 & 7,5 & 15,2 \\
\hline Pará & 88,2 & 31,9 & 1,16 & 6,5 & 7,7 & 13,4 \\
\hline Amapá & 97,2 & 28,1 & 1,47 & 7,3 & 8,8 & 12,4 \\
\hline Tocantins & 90,9 & 32,7 & 1,03 & 6,1 & 7,1 & 12,8 \\
\hline Maranhão & 84,3 & 39,5 & 1,05 & 6,2 & 7,2 & 12,5 \\
\hline Piauí & 91,5 & 31,0 & 1,19 & 6,4 & 7,6 & 11,7 \\
\hline Ceará & 90,9 & 29,4 & 1,42 & 6,7 & 8,1 & 12,5 \\
\hline Rio Grande do & 90,0 & 28,5 & 1,44 & 6,8 & 8,3 & 13,8 \\
\hline \multicolumn{7}{|l|}{ Norte } \\
\hline Paraíba & 91,8 & 26,2 & 1,16 & 5,7 & 6,9 & 10,8 \\
\hline Pernambuco & 95,3 & 31,7 & 1,30 & 6,5 & 7,8 & 12,3 \\
\hline Alagoas & 91,5 & 33,6 & 1,73 & 6,6 & 8,4 & 12,8 \\
\hline Sergipe & 95,1 & 26,0 & 1,39 & 6,5 & 7,9 & 8,9 \\
\hline Bahia & 88,5 & 30,2 & 1,46 & 7,1 & 8,5 & 12,2 \\
\hline Minas Gerais & 94,1 & 22,2 & 1,49 & 7,9 & 9,4 & 11,9 \\
\hline Espírito Santo & 98,1 & 24,8 & 1,26 & 6,7 & 8,0 & 10,2 \\
\hline Rio de Janeiro & 99,7 & 24,4 & 1,53 & 7,5 & 9,0 & 12,1 \\
\hline São Paulo & 99,0 & 21,1 & 1,43 & 7,9 & 9,3 & 12,1 \\
\hline Paraná & 98,5 & 25,4 & 1,30 & 7,1 & 8,4 & 10,7 \\
\hline Santa Catarina & 98,4 & 26,7 & 1,18 & 6,7 & 7,9 & 10,9 \\
\hline Rio Grande do Sul & 97,4 & 24,5 & 1,42 & 7,8 & 9,2 & 11,8 \\
\hline Mato Grosso do Sul & 97,8 & 27,5 & 1,06 & 6,6 & 7,7 & 12,2 \\
\hline Mato Grosso & 95,2 & 25,8 & 1,17 & 6,2 & 7,3 & 10,8 \\
\hline Goiás & 96,8 & 25,2 & 1,23 & 7,2 & 8,4 & 11,3 \\
\hline Distrito Federal & 100,0 & 26,0 & 1,55 & 8,1 & 9,6 & 11,0 \\
\hline Brasil & 94,8 & 27,3 & 1,35 & 7,2 & 8,5 & 11,9 \\
\hline
\end{tabular}

Fonte: * Sistema de Informações sobre Nascidos Vivos (SINASC) 2012, 2013 e 2014; ** Sistema de Informações sobre Mortalidade (SIM), 2013; *** SINASC, 2013.

fase (total de 4.591 casos) passaram por uma nova tentativa de relacionamento com o SINASC, utilizando o nome da mãe e a data do parto como variáveis-chave. Após as duas etapas de linkage, foram relacionados 23.057 casos, que correspondem a 95,3\% dos casos do banco do estudo Nascer no Brasil. Diferenças nas distribuições das variáveis consideradas no estudo entre os casos do estudo Nascer no Brasil que foram relacionados ao SINASC e os que não foram relacionados encontram-se no material suplementar (http://cadernos.ensp.fiocruz.br/site/public_site/arquivo/csp-2149-18-tabela-suple mentar_8607.pdf).

Os resultados de concordância de preenchimento das variáveis comuns aos dois bancos encontram-se na Tabela 2. Observam-se coeficientes kappa de concordância estatisticamente diferentes de zero para todas as variáveis testadas, bem como valor do nível de significância (valor de p) do teste quiquadrado de independência entre as variáveis sempre inferior a 0,001. Além disso, as distribuições marginais para todas as variáveis são muito semelhantes nas duas bases de dados. Contudo, os valores de kappa para algumas variáveis são próximos à unidade, como faixa de idade, peso ao nascer, tipo de 


\section{Figura 1}

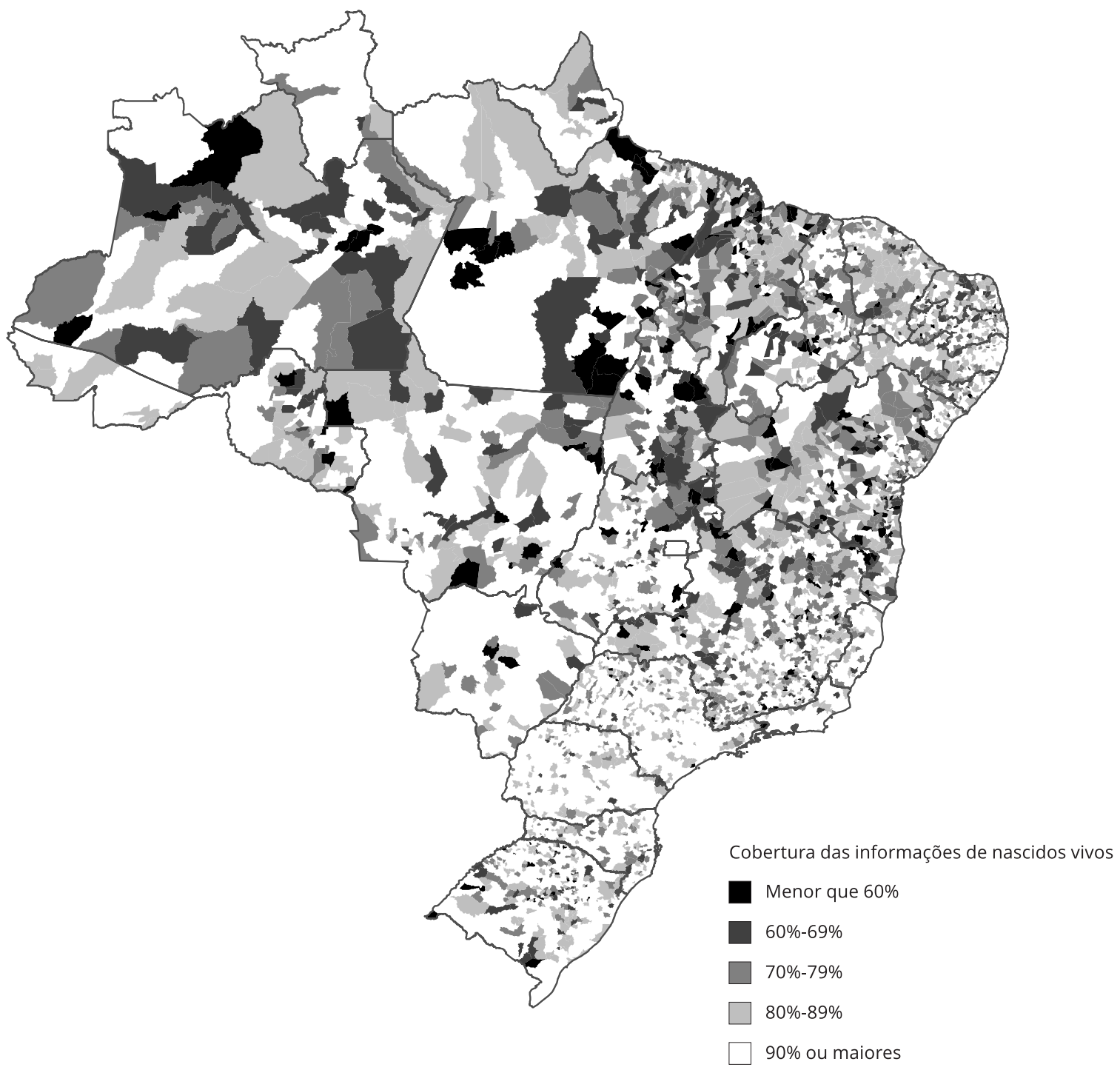

gestação, tipo de parto, Apgar no primeiro minuto, o que significa que as observações são classificadas exatamente nas mesmas categorias nas duas bases de dados. Já para outras variáveis, como anos de estudo e número de consultas pré-natal, os coeficientes estão entre 0,6 e 0,7, o que mostra concordância classificada como boa 13. A variável que mostrou pior concordância foi a idade gestacional, mensurada de diferentes formas no Nascer no Brasil e SINASC, com valor de kappa de 0,461 (IC95\%: 0,445-0,477), considerada uma concordância moderada 13 .

$\mathrm{Na}$ Figura 3, são apresentados os percentuais de prematuridade, de acordo com faixas de peso ao nascer por Grande Região, calculados por meio das informações do SINASC 2013. Os percentuais de prematuridade foram comparados ao padrão encontrado no estudo Nascer no Brasil. Observam-se as maiores diferenças na Região Norte. Para a primeira faixa de peso ao nascer $(<1.500 \mathrm{~g})$, o valor espe- 


\section{Figura 2}

Procedimento de linkage entre as bases de dados do estudo Nascer no Brasil e o Sistema de Informações sobre Nascidos Vivos, 2011-2012.

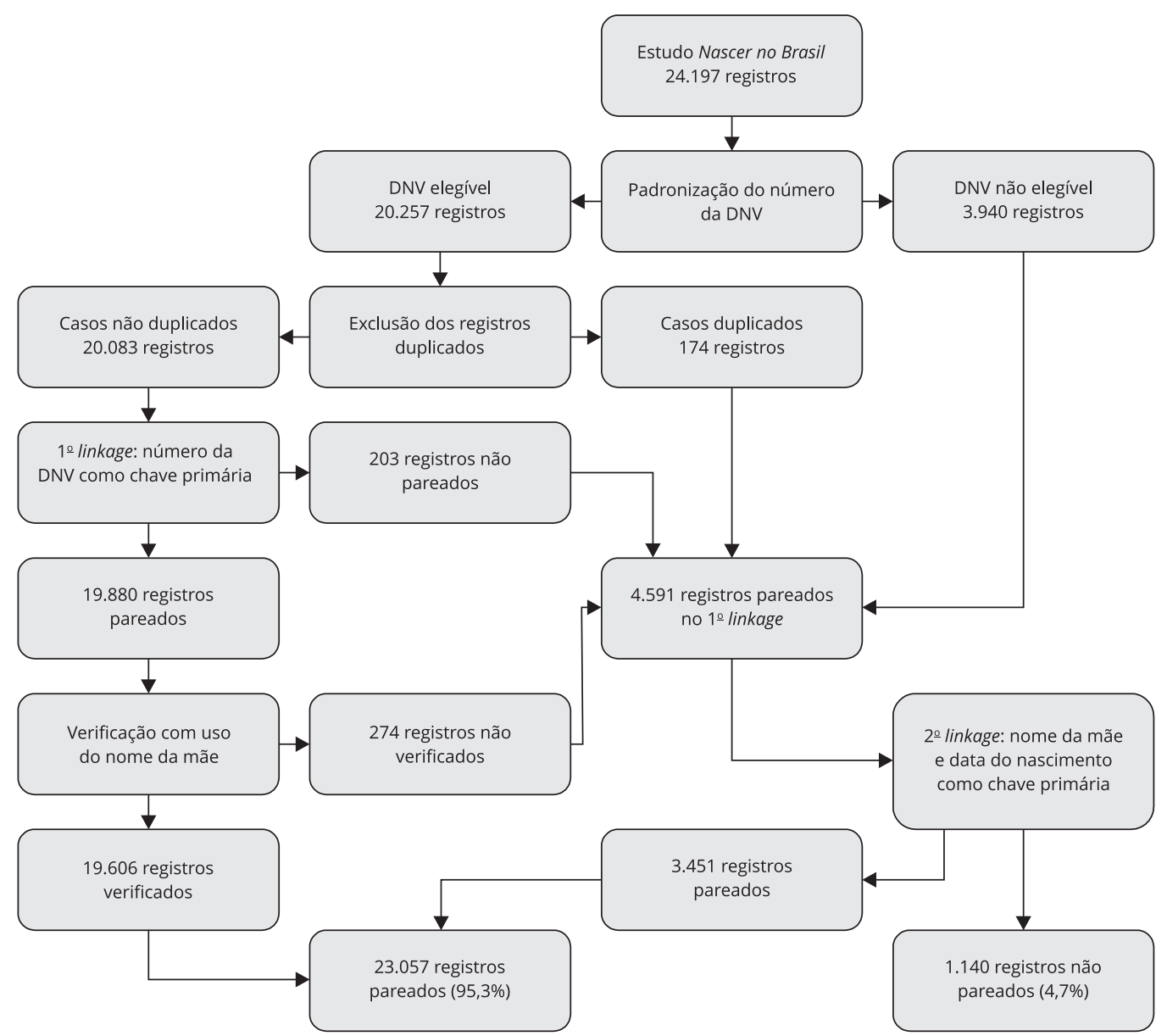

DNV: declaração de nascido vivo.

rado de percentual de prematuridade é de $98 \%$, mas na Região Norte, o percentual encontrado foi de $83 \%$, e na Região Nordeste, de 86\%. Para as categorias de maior peso ao nascer (3.000-3.999g e maior ou igual a $4.000 \mathrm{~g}$ ), as maiores diferenças foram encontradas nas mesmas regiões, com percentuais bem superiores aos esperados. A menor distância, estabelecida pela SQE entre os percentuais informados e esperados de acordo com o padrão, ocorreu na Região Sul (SQE = 126,7), seguida da Sudeste $(\mathrm{SQE}=166,6)$, e a maior distância correspondeu à Região Norte $(\mathrm{SQE}=581,2)$.

\section{Discussão}

Os resultados do presente estudo mostram cobertura das informações de nascidos vivos alta e homogênea, com abrangência de mais de $90 \%$ dos nascidos vivos na maioria das unidades da federação. Uma vez que o número de nascidos vivos é usado no cálculo de vários dos indicadores de desenvolvimento sustentável, o progresso alcançado na captação de nascidos vivos é um passo importante para a estimação dos indicadores de saúde materno-infantil ${ }^{1}$ com fidedignidade. 
Tabela 2

Distribuições de variáveis selecionadas nas bases de dados do estudo Nascer no Brasil e do SINASC (2011-2012) e coeficientes kappa de concordância entre os dados relacionados.

\begin{tabular}{|c|c|c|c|c|}
\hline Variável & Nascer no Brasil & SINASC & Kappa (IC95\%) & Valor de $p$ \\
\hline \multicolumn{5}{|c|}{ Idade da mãe (anos) } \\
\hline$<15$ & 0,9 & 0,9 & $0,938(0,935-0,942)$ & $<0,001$ \\
\hline $15-19$ & 18,1 & 18,3 & & \\
\hline $20-24$ & 28,2 & 28,0 & & \\
\hline $25-29$ & 24,4 & 24,3 & & \\
\hline $30-34$ & 17,8 & 17,9 & & \\
\hline $35-39$ & 8,6 & 8,7 & & \\
\hline $40-44$ & 1,8 & 1,8 & & \\
\hline $45+$ & 0,2 & 0,1 & & \\
\hline \multicolumn{5}{|c|}{ Anos de estudo da mãe } \\
\hline Nenhum & 1,4 & 0,8 & $0,680(0,671-0,689)$ & $<0,001$ \\
\hline $1-3$ & 3,7 & 4,1 & & \\
\hline $4-7$ & 21,4 & 23,6 & & \\
\hline $8-11$ & 59,2 & 56,2 & & \\
\hline $12+$ & 14,3 & 15,4 & & \\
\hline \multicolumn{5}{|c|}{ Peso ao nascer (g) } \\
\hline$<1.500$ & 1,6 & 1,6 & $0,942(0,939-0,946)$ & $<0,001$ \\
\hline $1.500-1.999$ & 1,9 & 2,0 & & \\
\hline $2.000-2.499$ & 5,8 & 6,0 & & \\
\hline $2.500-2.999$ & 24,0 & 24,2 & & \\
\hline $3.000-3.499$ & 40,7 & 40,8 & & \\
\hline 3.500-3.999 & 20,8 & 21,0 & & \\
\hline 4.000 & 5,2 & 4,5 & & \\
\hline \multicolumn{5}{|c|}{ Idade gestacional (em semanas) } \\
\hline$<27$ & 0,6 & 0,6 & $0,461(0,445-0,477)$ & $<0,001$ \\
\hline $28-31$ & 1,5 & 1,4 & & \\
\hline $32-36$ & 9,7 & 9,6 & & \\
\hline $37-41$ & 85,8 & 85,5 & & \\
\hline $42+$ & 2,4 & 2,9 & & \\
\hline \multicolumn{5}{|c|}{ Número de consultas de pré-natal } \\
\hline Nenhuma & 1,3 & 2,3 & $0,639(0,629-0,648)$ & $<0,001$ \\
\hline $1-3$ & 9,1 & 7,7 & & \\
\hline $4-6$ & 30,1 & 27,4 & & \\
\hline $7+$ & 59,5 & 62,6 & & \\
\hline \multicolumn{5}{|l|}{ Tipo de gestação } \\
\hline Única & 97,8 & 97,7 & $0,963(0,951-0,975)$ & $<0,001$ \\
\hline Gemelar ou + & 2,2 & 2,3 & & \\
\hline \multicolumn{5}{|l|}{ Tipo de parto } \\
\hline Vaginal & 46,4 & 47,8 & $0,919(0,913-0,924)$ & $<0,001$ \\
\hline Cesáreo & 53,6 & 52,2 & & \\
\hline \multicolumn{5}{|l|}{ Apgar 1o minuto } \\
\hline $0-3$ & 1,5 & 1,6 & $0,913(0,905-0,921)$ & $<0,001$ \\
\hline $4-6$ & 4,9 & 4,7 & & \\
\hline 7 & 6,2 & 6,7 & & \\
\hline 8-10 & 87,3 & 87,0 & & \\
\hline \multicolumn{5}{|l|}{ Apgar 5o minuto } \\
\hline $0-3$ & 0,3 & 0,4 & $0,855(0,832-0,879)$ & $<0,001$ \\
\hline $4-6$ & 0,7 & 0,7 & & \\
\hline 7 & 1,3 & 1,2 & & \\
\hline $8-10$ & 97,7 & 97,7 & & \\
\hline
\end{tabular}

IC95\%: intervalo de 95\% de confiança; SINASC: Sistema de Informações sobre Nascidos Vivos.

Fonte: Base relacionada dos dados do estudo Nascer no Brasil 9 com o SINASC (2011-2012). 


\section{Figura 3}

Avaliação da consistência das informações de idade gestacional do Sistema de Informações sobre Nascidos Vivos 2014 , de acordo com o peso ao nascer, por grande região, de acordo com o padrão nacional, Brasil.

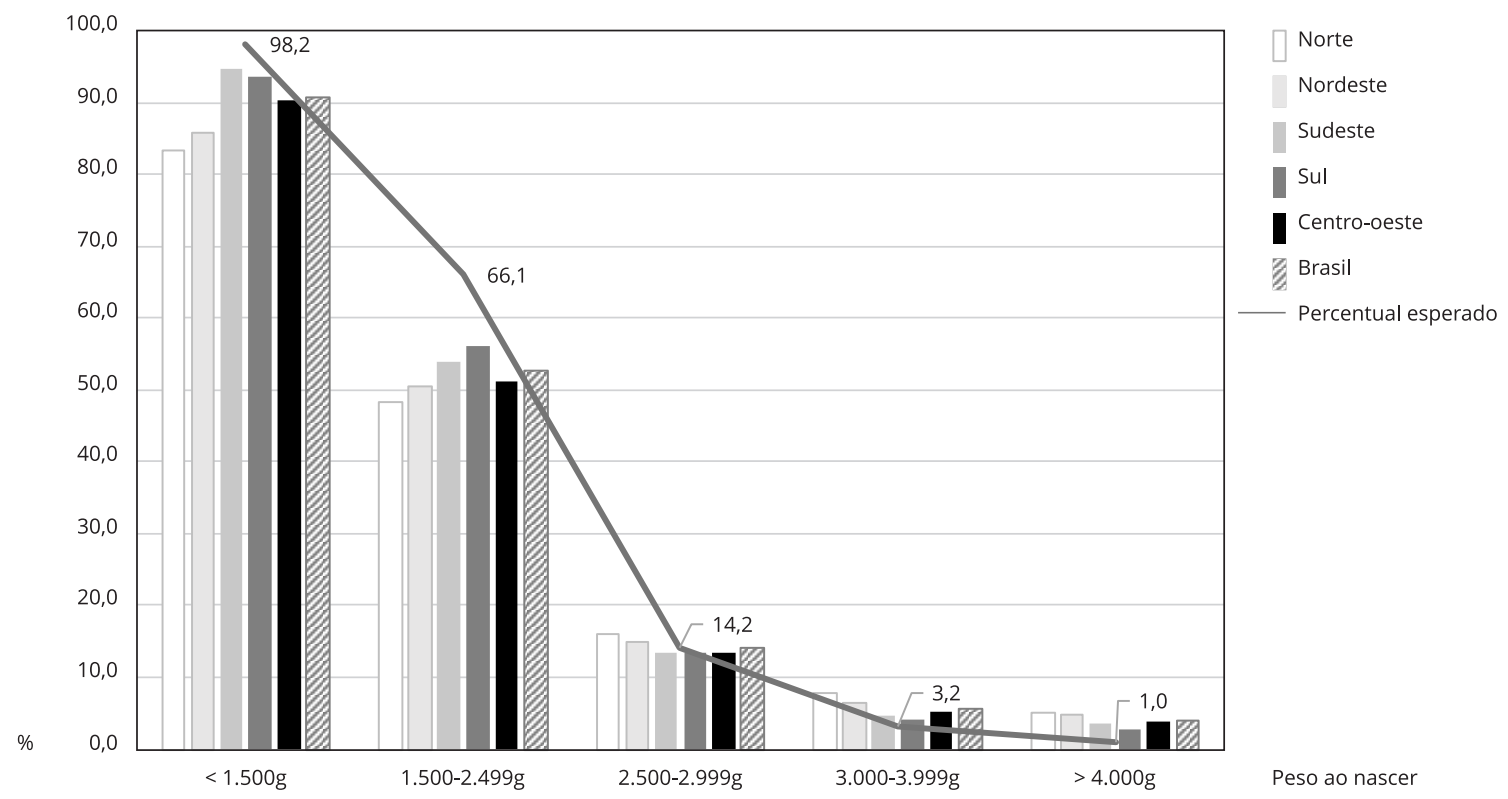

Reconhecidamente, o decréscimo da subenumeração de nascidos vivos ocorreu em todas as regiões, mesmo naquelas com maior precariedade das informações vitais 14,15. Resultados de investigação de busca ativa de óbitos e nascimentos realizada na Amazônia Legal e no Nordeste mostraram crescimento importante na cobertura das informações de nascidos vivos no período 2000-2010 16.

No presente trabalho, para calcular a cobertura das informações, propôs-se método simplificado, que pode ser utilizado no nível de município, por meio de informações secundárias, disponíveis anualmente no portal de dados do Ministério da Saúde. O método consiste em comparar o número informado de nascidos vivos com o número de nascidos vivos estimado pela população de menores de um ano, e vem sendo utilizado, igualmente, em países europeus, como a Dinamarca 17. Sua grande vantagem está em poder alertar de maneira simplificada sobre a falta de confiabilidade das informações de nascidos vivos. A aplicação do método para os municípios brasileiros mostrou que, apesar do nítido avanço, alguns municípios ainda têm subenumeração das informações de nascidos vivos em nível maior do que 20\%, o que afeta a construção de vários indicadores de saúde materno-infantil no nível local 18,19. Conforme apontado em estudos anteriores, a falta de informações adequadas dificulta traçar o perfil de saúde no município e subsidiar as políticas locais de saúde 20,21.

No que se refere à qualidade do preenchimento das informações do SINASC, a comparação com os dados coletados no estudo Nascer no Brasil mostrou, em geral, alto nível de concordância, principalmente se a variável é definida da mesma forma nas duas bases de dados. No caso das variáveis sócio-demográficas da mãe, por exemplo, para a idade, o grau de concordância é maior do que 90\%, mas no tocante ao nível de instrução, o grau de concordância não é tão alto porque essa variável é mensurada pelos anos de estudo, no SINASC, ao passo que, no estudo Nascer no Brasil, é baseada na informação sobre o último grau cursado. O mesmo pode ser dito para estado civil. Diferentemente do estudo Nascer no Brasil, a categoria "união estável/vive com companheiro" não é incluída no SINASC, e não houve possibilidade de verificar a qualidade de preenchimento dessa variável.

Em relação às variáveis da criança, como peso ao nascer e Apgar no primeiro e no quinto minutos, o coeficiente de concordância alcançou níveis excelentes. Da mesma forma, a concordância das variá- 
veis relativas ao tipo de parto (normal/cesáreo) e ao tipo de gestação (única/gemelar) foi quase perfeita. Esses resultados já tinham sido comprovados anteriormente por meio da análise do relacionamento das informações referentes ao óbito infantil no Sistema de Informações sobre Mortalidade (SIM) com as informações relativas ao nascimento e às características da mãe da criança falecida, obtidas pelo SINASC 22,23,24. Ressalta-se, porém, que a comparação das informações do SINASC com o SIM consiste em analisar, por vezes, informações do mesmo prontuário, tendo em vista a grande proporção de óbitos infantis que ocorrem próximos ao parto. A validação por meio de resultados, com coleta de dados primários, como os do estudo Nascer no Brasil, reafirma a qualidade das informações do SINASC.

Um achado que chamou a atenção foi o coeficiente de concordância inferior a 70\% para o número de consultas pré-natal, quando se comparam os dados do SINASC aos do estudo Nascer no Brasil. Análise detalhada do cruzamento das gestantes presentes nas duas bases de dados mostrou que, entre aquelas sem consulta pré-natal no SINASC, 68,8\% tinham uma ou mais consultas de acordo com o Nascer no Brasil. Outro marcador importante de avaliação da assistência à gestação, o trimestre de início do atendimento pré-natal, apresenta muitas falhas de preenchimento no SINASC, com $41 \%$ dos casos sem informação para essa variável, apesar de apenas 1,8\% desses casos não ter a informação preenchida no estudo Nascer no Brasil.

No que se refere à idade gestacional, no SINASC, a informação é coletada em semanas gestacionais, com base, prioritariamente, na data da última menstruação (DUM). Contudo, na falta de informação sobre a DUM, o registro da idade gestacional no sistema de nascidos vivos é realizado a partir de outros métodos de aferição (exame físico ou ultrassonografia). Já no Projeto Nascer, foram propostas várias formas para mensurar idade gestacional: DUM relatada pela puérpera e DUM anotada no prontuário materno, autorrelato da idade gestacional na entrevista com a puérpera e resultado da ultrassonografia entre sete e vinte semanas de gestação 25. Comparando-se os percentuais de prematuridade nas duas bases de dados, os valores são bem próximos. De fato, a partir de 2011, a informação de idade gestacional no SINASC passou a ser coletada como variável contínua, resultando na maior adequação do indicador de prematuridade 26 .

Contudo, quando são analisadas as medidas de idade gestacional nas duas bases de dados, independentemente do método de mensuração, as discrepâncias são grandes e o grau de concordância mostrou-se inferior a 50\%. Esses achados corroboram análises anteriores de validação do SINASC, que mostraram a baixa concordância entre a proporção de nascidos vivos prematuros no sistema e a obtida em pesquisas de base populacional 26 . Além disso, o indicador que descreve as inconsistências, medido pela soma dos quadrados das diferenças entre os percentuais de prematuridade informados e esperados por faixa de peso ao nascer, teve o valor mais alto na Região Norte e o menor na Região Sul, apontando para desigualdades geográficas na mensuração da idade gestacional.

O problema do SINASC reside, assim, em obter uma informação válida da idade gestacional, que possa ser utilizada em todas as UF. Embora a ultrassonografia precoce seja considerada o método mais preciso para estimar a idade gestacional 25, a informação baseada na DUM é o método recomendado pela Organização Mundial da Saúde (OMS) 27, devido à sua elevada acessibilidade e ao baixo custo. Porém, a idade gestacional baseada na DUM apresenta falhas decorrentes de variações na duração do ciclo menstrual e de dificuldades de memória, associadas, em geral, ao baixo nível de escolaridade das gestantes 28,29.

Entre as limitações deste estudo, no que se refere à análise de cobertura das informações do SINASC, estão as estimativas da população menor de um ano fornecidas pelo IBGE, uma vez que erros nas estimativas populacionais podem acarretar problemas no cálculo da cobertura das informações de nascidos vivos. Já no que diz respeito à avaliação da qualidade dos dados, destaca-se que as informações do SINASC foram comparadas às do estudo Nascer no Brasil, que não tem abrangência nacional. Os dados do inquérito são representativos de nascidos vivos em maternidades com 500 partos ou mais, e não incluem os nascidos vivos em maternidades pequenas ou os nascdido vivos em domicílios, o que pode ter afetado as medidas de concordância. Além disso, tivemos algumas variáveis definidas de forma diferente entre o estudo Nascer no Brasil e o SINASC, como estado civil e raça, impossibilitando avaliar o preenchimento desses campos. Da mesma forma, a falta de informação em determinadas variáveis da base do Projeto Nascer, que foram utilizadas como chave para o "linkage" das bases de dados, como por exemplo o número da DNV, prejudicou a identificação dos pares na base do SINASC. 


\section{Conclusão}

No presente estudo, foi apresentado método que pode ser replicado, facilmente, por outros pesquisadores e gestores ou técnicos das secretarias de saúde, com base em dados disponíveis publicamente. Mostrou-se que a cobertura das informações de nascidos vivos é adequada para grande parte dos municípios brasileiros, embora níveis inferiores a $60 \%$ ainda sejam encontrados em municípios localizados nas áreas mais pobres do país. Quanto à qualidade dos dados do SINASC, verificou-se que o maior problema se refere à idade gestacional, com informações menos fidedignas ocorrendo, igualmente, nas regiões menos desenvolvidas do país. Tendo em vista que informações imprecisas sobre a idade gestacional repercutem na promoção, prevenção e atenção à saúde da criança, essas questões precisam ser abordadas para melhorar a capacidade dos níveis locais de planejar e avaliar as políticas de saúde destinadas a melhorar a saúde das mães e dos bebês. Medidas não confiáveis podem ser decisivas na exclusão de grupos que poderiam ser os maiores beneficiários de intervenções e políticas públicas.

\section{Colaboradores}

C. L. Szwarcwald e M. C. Leal contribuíram no projeto do estudo, análise e interpretação dos dados; redação, revisão, leitura e aprovação da versão final do manuscrito. A. P. Esteves-Pereira, W. S. Almeida, P. G. Frias, G. N. Damacena, P. R. B. Souza Júnior, N. M. Rocha e P. M. H. Mullachery colaboraram na análise dos dados, redação, revisão, leitura e aprovação da versão final do manuscrito.

\section{Informações adicionais}

ORCID: Célia Landmann Szwarcwald (0000-00027798-2095); Maria do Carmo Leal (0000-00023047-515X); Ana Paula Esteves-Pereira (00000002-0236-2043); Wanessa da Silva de Almeida (0000-0002-5164-8603); Paulo Germano de Frias (0000-0003-4497-8898); Giseli Nogueira Damacena (0000-0002-7059-3353); Paulo Roberto Borges de Souza Júnior (0000-0002-8142-4790); Narayani Martins Rocha (0000-0002-6943-01094); Priscila Melissa Honorato Mullachery (0000-0003-47583875).

\section{Agradecimentos}

Agradecemos aos coordenadores regionais e estaduais, supervisores, coletores de dados e a todos os funcionários das unidades de saúde participantes do estudo Nascer no Brasil. Agradecemos também às mães que participaram e tornaram este estudo possível.

\section{Referências}

1. AbouZahr C, Savigny D, Mikkelsen L, Setel PW, Lozano R, Nichols E, et al. Civil registration and vital statistics: progress in the data revolution for counting and accountability. Lancet 2015; 386:1373-85.

2. Szwarcwald CL, Leal MC, Andrade CLT, Souza Jr. PRB. Infant mortality estimation in Brazil: what do Ministry of Health data on deaths and live births say? Cad Saúde Pública 2002; 18:1725-36.

3. Jorge MHM, Laurenti R, Gotlieb SL. Quality analysis of Brazilian vital statistics: the experience of implementing the SIM and SINASC systems. Ciênc Saúde Colet 2007; 12:643-54.

4. Theme Filha MM, Gama SGN, Cunha CB, Leal MC. Confiabilidade do Sistema de Informações sobre Nascidos Vivos Hospitalares no Município do Rio de Janeiro, 1999-2001. Cad Saúde Pública 2004; 20 Suppl 1:S83-91.

5. Guimarães EAA, Hartz ZMA, Loyola Filho AI, Meira AJ, Luz ZMP. Avaliação da implantação do Sistema de Informação sobre Nascidos Vivos em municípios de Minas Gerais, Brasil. Cad Saúde Pública 2013; 29:2105-18.

6. Silva LP, Moreira CM, Amorim MH, Castro DS, Zandonade E. Evaluation of the quality of data in the Live Birth Information System and the Information System on Mortality during the neonatal period in the state of Espírito Santo, Brazil, between 2007 and 2009. Ciênc Saúde Colet 2014; 19:2011-20.

7. Silva RS, Oliveira CM, Ferreira DKS, Bonfim CV. Avaliação da completitude das variáveis do Sistema de Informações sobre Nascidos Vivos - SINASC - nos Estados da região Nordeste do Brasil, 2000 e 2009. Epidemiol Serv Saúde 2013; 22:347-52. 
8. Pereira CCB, Vidal SA, Carvalho PI, Frias PG. Evaluation of data quality from the information system on live births in 1997-1998. Rev Bras Saúde Mater Infant 2013; 13:39-49.

9. Leal MC, Silva AA, Dias MA, Gama SG, Rattner D, Moreira ME, et al. Birth in Brazil: national survey into labour and birth. Reprod Health 2012; 9:15.

10. Lima CRA, Schramm JMA, Coeli CM, Silva MEM. Revisão das dimensões de qualidade dos dados e métodos aplicados na avaliação dos sistemas de informação em saúde. Cad Saúde Pública 2009; 25:2095-2109.

11. Frias PG, Szwarcwald CL, Souza Junior PRB, Almeida WS, Lira PI. Correcting vital information: estimating infant mortality, Brazil, 20002009. Rev Saúde Pública 2013; 47:1048-58.

12. Interagency Health Information Network. Basic indicators for health in Brazil: concepts and applications. http://tabnet.datasus.gov.br/tab data/livroidb/2ed/indicadores.pdf (acessado em 20/Abr/2017)

13. Vasconcellos MTL, Silva PLN, Pereira APE, Schilithz AOC, Souza Junior PRB, Szwarcwald CL. Desenho da amostra Nascer no Brasil: Pesquisa Nacional sobre Parto e Nascimento. Cad Saúde Pública 2014; 30 Suppl 1:S49-58.

14. Frias PG, Szwarcwald CL, Lira PIC. Avaliação dos sistemas de informações sobre nascidos vivos e óbitos no Brasil na década de 2000. Cad Saúde Pública 2014; 30:2068-80.

15. Pedraza DF. Quality of the Information System on Live Births/SINASC: a critical analysis of published studies. Ciênc Saúde Colet 2012; 17:2729-37.

16. Szwarcwald CL, Frias PG, Souza Júnior PRB, Almeida WS, Morais Neto OL. Correction of vital statistics based on a proactive search of deaths and live births: evidence from a study of the North and Northeast regions of Brazil. Popul Health Metr 2014; 12:16.

17. Schmidt M, Pedersen L, Sørensen HT. The Danish civil registration system as a tool in epidemiology. Eur J Epidemiol 2014; 29:541-9.

18. Blencowe H, Calvert C, Lawn JE, Cousens S, Campbell OM. Measuring maternal, foetal and neonatal mortality: challenges and solutions. Best Pract Res Clin Obstet Gynaecol 2016; 36:14-29.

19. Grove J, Claeson M, Bryce J, Amouzou A, Boerma T, Waiswa P, et al. Maternal, newborn, and child health and the Sustainable Development Goals - a call for sustained and improved measurement. Lancet 2015; 386:1511-4.
20. Gabriel GP, Chiquetto L, Morcillo AM, Ferreira MC, Bazan IG, Daolio LD, et al. Evaluation of data on live birth certificates from the Information System on Live Births (SINASC) in Campinas, São Paulo, 2009. Rev Paul Pediatr 2009; 32:183-8.

21. Rodrigues M, Bonfim C, Portugal JL, Frias PG, Gurgel IG, Costa TR, et al. Spatial analysis of infant mortality and the adequacy of vital information: a proposal for defining priority areas. Ciênc Saúde Colet 2014; 19:2047-54.

22. Gaiva MA, Fujimori E, Sato AP. Neonatal mortality in infants with low birth weight. Rev Esc Enferm USP 2014; 48:778-86.

23. Kassar SB, Melo AM, Coutinho SB, Lima MC, Lira PI. Determinants of neonatal death with emphasis on health care during pregnancy, childbirth and reproductive history. J Pediatr (Rio J.). 2013; 89:269-77.

24. Morais Neto OL, Barros MBA. Risk factors for neonatal and post-neonatal mortality in the Central-West region of Brazil: linkage between live birth and infant death data banks. Cad Saúde Pública 2000; 16:477-85.

25. Pereira APE, Leal MC, Gama SGN, Domingues RMSM, Schilithz AOC, Bastos MH. Determinação da idade gestacional com base em informações do estudo Nascer no Brasil. Cad Saúde Pública 2014; 30 Suppl 1:S59-70.

26. Silveira MF, Matijasevich A, Horta BL, Bettiol $\mathrm{H}$, Barbieri MA, Silva AA, et al. Prevalence of preterm birth according to birth weight group: a systematic review. Rev Saúde Pública 2013; 47:992-1003

27. World Health Organization. Home-based maternal records. Guidelines for development, adaptation and evaluation. Geneva: World Health Organization; 1994.

28. Hoffman CS, Messer LC, Mendola P, Savitz DA, Herring AH, Hartmann KE. Comparison of gestational age at birth based on last menstrual period and ultrasound during the first trimester. Paediatr Perinat Epidemiol 2008; 22:587-96

29. Wingate MS, Alexander GR, Buekens P, Vahratian A. Comparison of gestational age classifications: date of last menstrual period vs. clinical estimate. Ann Epidemiol 2007; 17:425-30. 


\section{Abstract}

This study is a quantitative and qualitative assessment of data from the Brazilian Information System on Live Births (SINASC) in Brazil. Coverage of the data by municipality was estimated as the ratio between reported and estimated live births. Data quality in the SINASC was assessed via probabilistic linkage with the database from the Birth in Brazil study, 2011-2012, and kappa coefficients of agreement were calculated. In 2013, data coverage was high and homogeneous in all states of Brazil. However, the analysis according to municipalities (counties) showed greater spatial heterogeneity. As for completeness of information in SINASC, kappa coefficients were statistically different from zero for all the tested variables ( $p$ $<0.001$ ), and marginal distributions of all the variables were similar in the two databases. Gestational age was the variable with the worst agreement, with a kappa value of 0.461 . The indicator that describes the inconsistencies, measured by the sum of the square of the differences between the reported and expected prematurity rates by birthweight bracket, showed the highest value in the North of Brazil and the lowest in the South, pointing to geographic inequalities in measurement of gestational age.

Live Births; Health Information Systems; Quality Management

\section{Resumen}

En este trabajo, se evalúa cuantitativa y cualitativamente la información del Sistema de Información sobre Nacidos Vivos (SINASC) de Brasil. La cobertura de la información por municipio se estimó por la razón entre nacidos vivos informados y estimados. Para la evaluación de la calidad de la información del SINASC, se efectuó la relación del sistema con la base de datos del estudio Nacer no Brasil, 2011-2012 y se estimaron los coeficientes kappa de concordancia. En 2013, la cobertura de la información fue alta y homogénea en todas las unidades de la Federación. No obstante, el análisis por municipio brasileño presentó mayor heterogeneidad espacial. En cuanto a la calidad de cumplimentación de la información del SINASC, los coeficientes kappa de concordancia fueron estadísticamente diferentes de cero para todas las variables probadas $(p<0,001)$ y las distribuciones marginales para todas las variables consideradas fueron semejantes en las dos bases de datos. La edad gestacional fue la variable que mostró peor concordancia, con valor de kappa de 0,461. El indicador que describe las inconsistencias, medido por la suma del cuadrado de las diferencias entre los porcentajes de prematuridad informados y esperados por franja de peso al nacer, tuvo el valor más alto en la región Norte y el menor en la Sur, apuntando desigualdades geográficas en la medición de la edad gestacional.

Nacimiento Vivo; Sistemas de Información en Salud; Gestión de la Calidad
Recebido em 13/Nov/2018

Versão final reapresentada em 29/Abr/2019

Aprovado em 07/Mai/2019 